\title{
Evolution of Pulse and Periodic Elastic Waves in Media with Quadratically-Bimodular Nonlinearity
}

\author{
V.E. Nazarov, S. B. Kiyashko
}

On the basis of the elastic contact model of rough surfaces of solids, a quadraticallybimodular equation of state for micro-inhomogeneous media containing cracks is derived. A study is made of the propagation of elastic single unipolar pulse perturbations and bipolar periodic waves in such media. Exact analytical solutions that describe the evolution of initially triangular pulses and periodic sawtooth waves are obtained. A numerical and graphical analysis of the solutions is also carried out.

Keywords: elastic contact, quadratically-bimodular nonlinearity, pulse perturbation, periodic waves

\section{Introduction}

The theory of nonlinear wave processes in homogeneous ideal media with a quadratic nonlinearity is developed in sufficient detail [1-4]. In the process of propagation in such media of longitudinal elastic waves (called simple [1-4] ones), first of all, their leading or rear (depending on the sign of the medium nonlinearity parameter) wave front begins to become steeper. Then, in the profile of such waves, an ambiguity or "overlap" is formed [1-4]. Due to the physical unrealizability of the "overlap", a break-shock front is artificially introduced into the wave profile. This makes it possible to eliminate the ambiguity of the profile and leads to nonlinear absorption of the wave, while its amount of motion is conserved and wave energy decreases [1-4].

Since recently, acoustics has been paying increasing attention to the study of nonlinear wave processes in micro-inhomogeneous solids [5-7]. The acoustic nonlinearity of such media is anomalously high in comparison with weakly nonlinear homogeneous media that are described by five-constant elasticity theory [3]. It is determined by nonlinear defects of their structure: dislocations, cavities, cracks, grain contacts, etc. The equations of state for micro-inhomogeneous solids are often described by a nonanalytic continuous (but nonsmooth and nondifferentiable)

Received March 20, 2018

Accepted June 28, 2018

Veniamin E. Nazarov

v.e.nazarov@appl.sci-nnov.ru

Sergey B. Kiyashko

sergey-kiyashko@mail.ru

Institute of Applied Physics RAS

ul. Ulyanova 46, Nizhniy Novgorod, 603950, Russia

RUSSIAN JOURNAL OF NONLINEAR DYNAMICS, 2018, 14(3), 331-342 
function. The patterns of propagation and evolution of elastic waves in media with nonanalytic and quadratic (analytical) nonlinearities [1-4] differ qualitatively [7] from each other. This feature can be used for diagnostics and nondestructive testing of micro-inhomogeneous media. This is also facilitated by the fact that the nonlinear acoustic properties of such media are more sensitive to the presence of defects in them than linear ones. The class of media with nonanalytic nonlinearity includes so-called different-modules (or bimodal) media. They are characterized by different modules $E_{1}$ and $E_{2}$ elasticity, respectively, under tension and compression. A rather wide class of media possesses a modular property: some polymers, composite and structural materials and soils, as well as solids containing cracks [8]. Therefore, the study of nonlinear wave processes in such media is of scientific and practical interest. From a scientific point of view, this is related to the identification of regularities of nonlinear wave processes in media with nonanalytic nonlinearity. Practical interest is due to the possibility of creating effective methods for diagnosing the structure of materials.

The process of propagation of longitudinal elastic waves in bimodular media has been studied by many researchers [7-18]. They assumed that the equation of state of such media has the form

$$
\sigma(\varepsilon)=\left\{\begin{array}{l}
E_{1} \varepsilon, \quad \varepsilon \geqslant 0, \\
E_{2} \varepsilon, \quad \varepsilon \leqslant 0
\end{array}=E_{0}[\varepsilon-\gamma|\varepsilon|],\right.
$$

where $\sigma$ and $\varepsilon$ are longitudinal stress and strain, $E_{0}=\frac{E_{1}+E_{2}}{2}, \gamma=\frac{E_{2}-E_{1}}{E_{1}+E_{2}}$ is the parameter of the bimodular nonlinearity, $|\gamma| \ll 1,|\varepsilon| \ll 1$.

In such bimodular media, the nonlinear propagation regime occurs only for bipolar waves, while unipolar perturbations propagate linearly with constant but different velocities (depending on their polarity) [13]. The nonlinear distortion of a bipolar initially harmonic wave occurs in this way: already at an arbitrarily small distance from the radiator, an ambiguity or "overlap" is formed at each wave period which is eliminated by introducing a shock (or shock front) [13] in the wave profile. It can be seen from Eq. (1.1) that the medium bimodularity is manifested at an arbitrarily small positive and negative longitudinal stresses and strains. Therefore, the bilinear function $\sigma=\sigma(\varepsilon)$ undergoes a break at a point $\varepsilon=0, \sigma=0$. However, for real bimodular media, for small $\sigma$ and $\varepsilon$, the dependence should be smooth without the break [8]. Such a property of bimodular media is described by the following equation of state with a quadratically-bimodular nonlinearity:

$$
\sigma(\varepsilon)=E_{0}\left(\varepsilon-\frac{\gamma_{1} \varepsilon^{2}}{2\left(1+\gamma_{0}|\varepsilon|\right)}\right)
$$

where $\gamma_{1}$ and $\gamma_{0}$ are the nonlinearity parameters, $\left|\gamma_{1} \varepsilon\right| \ll 1,|\varepsilon| \ll 1, \frac{\left|\gamma_{1} \varepsilon\right|}{2\left(1+\gamma_{0}|\varepsilon|\right)} \ll 1$, $\left|\gamma_{1}\right| / \gamma_{0} \ll 1, \gamma_{0} \geqslant 0$. At $\gamma_{0}|\varepsilon| \ll 1$, using Eq. (1.2), we obtain an equation with quadratic elastic nonlinearity; and at $\gamma_{0}|\varepsilon| \gg 1$, an equation with bimodular nonlinearity (except for a small area $\left.|\varepsilon|<\gamma_{0}^{-1}\right): \sigma(\varepsilon) \cong E_{0}(\varepsilon-\gamma|\varepsilon|)$, where $\gamma=\gamma_{1} / 2 \gamma_{0}$. For media described by the equation of state (1.2), the nonlinear propagation regime takes place for both bipolar waves and unipolar perturbations.

In this paper, we study the propagation of elastic single unipolar pulse perturbations and bipolar periodic waves in the media with quadratically-bimodular (nonanalytic) nonlinearity. In Section 2, on the basis of the crack model which is an elastic contact of rough surfaces of solids [21], we obtained the quadratically-bimodular equation of state for a micro-inhomogeneous medium with cracks. In Section 3, the nonlinear wave equation was obtained for simple strain waves. In Sections 4 and 5, analytical exact solutions describing the propagation and 
evolution of an initially unipolar triangular pulse perturbation and bipolar periodic sawtooth wave in such media were obtained. In the same sections, a numerical and graphical analysis of the solutions was carried out. In Section 6, there are differences in the patterns of propagation of a single unipolar perturbation and a periodic bipolar wave.

\section{Quadratic-bimodular state equation of solid containing cracks}

In this section, we will obtain a quadratically-bimodular equation of state (1.2) for a medium with cracks. Let us consider a nonlinear crack model which is the elastic contact of rough surfaces of linear ideally elastic solids pressed against each other under the action of internal stresses of the material surrounding the crack [21]. We will assume that the contact has the shape of a circle radius $R$. As is well known [22], when contacting elastic bodies without friction, the contact stresses depend only on the relative profile of the surfaces. Therefore, in calculating the elastic properties, the contact in the crack can be replaced by the contact of a planar rigid surface (1) with an equivalent body having an initial profile corresponding to the gap between undeformed rough surfaces (Fig. 1). The equivalent rough surface will be characterized by the distribution function $W=W(h)$ of the heights of the tops measured from the zero surface (2). In this connection, the composition $W(h) d h$ should be considered as the number of vertices with heights $h$ up to $h+d h$ per nominal crack area $S=\pi R^{2}$. Consider a contact in which under stress $\sigma$ the distance from the zero surface (2) to the hard surface (1) becomes equal to $d$, while the irregularities with the heights of the tops initially exceeding $d$ are elastically deformed and become flat. The force $f$ required to compress each irregularity depends on the displacement $\delta=h-d$ of the crack surfaces and their type. We will assume that the unevenness of crack surfaces has the form

- an obtuse cone with an opening angle $2 \psi \cong \pi$;

- parts of the sphere of radius $c$;

- a circular cylinder of radius $b$.

The force $f(\delta)$ required to compress one such unevenness is determined by the following expressions:

- for the cone: $f(\delta)=(\pi / 2) \operatorname{tg} \psi E^{*} \delta^{2}$,

- for a part of the sphere:

$$
f(\delta)=(16 c / 9)^{1 / 2} E^{*} \delta^{3 / 2},
$$

- for a circular cylinder: $f(\delta)=4 b E^{*} \delta$,

where $E^{*}=E / 2\left(1-\nu^{2}\right), E$ is Young's modulus, and $\nu$ is Poisson's ratio.

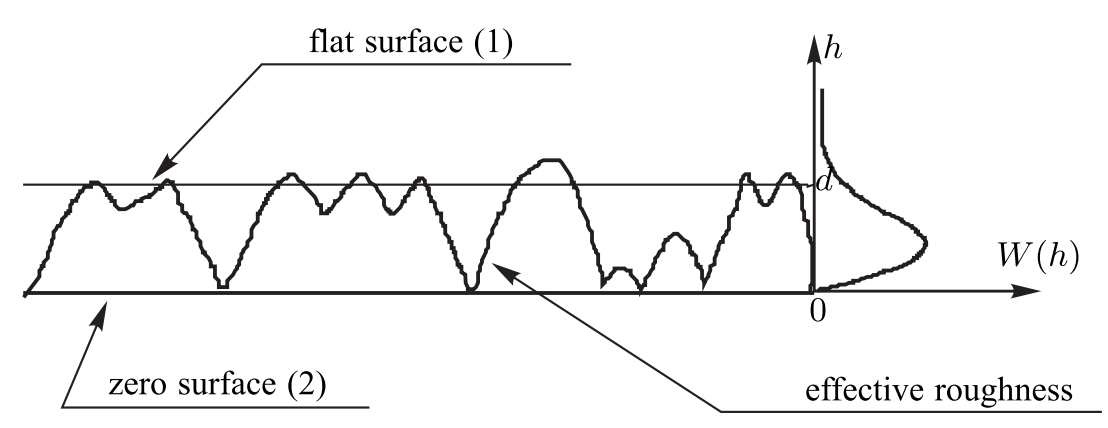

Fig. 1. Elastic contact of rough and flat surfaces. 
To find the dependence of the total stress $\sigma_{1}$ on the displacement $d$, we sum the forces for all the irregularities in contact, i.e., those irregularities that have a height $h \geqslant d[20]$ :

$$
\sigma_{1}(d)=\int_{d}^{\infty} f(h-d) W(h) d h .
$$

Assuming in (2.2) $W(h)=\frac{n}{\pi R^{2}} \frac{h}{h_{s}^{2}} \exp \left(-\frac{h}{h_{s}}\right), h_{s}=\sqrt{2} h_{0}, h_{0}$ is the effective height of the irregularities of each rough crack surface, $n$ is the number of vertices on the nominal area of the crack surface $S=\pi R^{2}$, we obtain

$$
\sigma_{1}(d)=E_{1}\left[d+m h_{s}\right] \exp \left(-\frac{d}{h_{s}}\right)
$$

where $m=3, E_{1}=\frac{\pi n h_{s} E^{*} \operatorname{tg} \psi}{\pi R^{2}}$ for cracks with cone-shaped roughness,

$$
\begin{aligned}
& m=5 / 2, E_{1}=\left(\pi c h_{s}\right)^{1 / 2} \frac{n E^{*}}{\pi R^{2}} \text { for cracks with spherical roughness, } \\
& m=2, E_{1}=\frac{4 n b E^{*}}{\pi R^{2}} \text { for cracks with cylindrical roughness. }
\end{aligned}
$$

Next, note that the contact is under the influence of static compressive stress $\sigma_{0}$, determined by the elasticity of the crack. This tension can be found by equating the expression for the volume $V_{0}$ of an open crack with smooth surfaces to the volume $V_{0}$ of a round crack with rough surfaces whose distance is equal to $d_{0}$. The normal displacements $U_{0}(r)$ of the original plane crack (thin cut) in a solid radius $R$ under the action of tensile stress $\sigma_{0} \geqslant 0$ are described by the expression [23]:

$$
U_{0}(r)=4\left(1-\nu^{2}\right)\left[R^{2}-r^{2}\right]^{1 / 2} \sigma_{0} / \pi E
$$

and its volume is

$$
V_{0}\left(d_{0}\right)=16\left(1-\nu^{2}\right) R^{3} \sigma_{0} / 3 E=\pi R^{2} d_{0},
$$

where $r$ is the radial coordinate in the plane of the crack.

From Eq. (2.5) we find

$$
\sigma_{0}\left(d_{0}\right)=3 \pi E d_{0} / 16\left(1-\nu^{2}\right) R .
$$

In the state of equilibrium of the crack, the compressive stress $\sigma_{0}\left(d_{0}\right)$ will be compensated by the tensile stress $\sigma_{1}\left(d_{0}\right)$ :

$$
\frac{3 \pi E d_{0}}{16\left(1-\nu^{2}\right) R}=E_{1}\left[d_{0}+m h_{s}\right] \exp \left(-\frac{d_{0}}{h_{s}}\right) .
$$

This transcendental equation determines the static distance $d_{0}$ between the midline of the rough and even surfaces of the contact in question. When an external stress $\sigma_{n}=\sigma$, normal to its surfaces, is applied to a crack, the distance between them will vary by an amount $\sigma(\widetilde{d})=\sigma_{0}\left(d_{0}+\right.$ $+\widetilde{d})-\sigma_{1}\left(d_{0}+\widetilde{d}\right)$ such that the following relationship is satisfied:

$$
\sigma(\widetilde{d})=\frac{3 \pi E\left(d_{0}+\widetilde{d}\right)}{16\left(1-\nu^{2}\right) R}-E_{1}\left[d_{0}+\widetilde{d}+m h_{s}\right] \exp \left(-\frac{d_{0}+\widetilde{d}}{h_{s}}\right) .
$$

From this equation it follows that it is possible to distinguish three different stages of deformation of the crack, i.e., three various dependences $\sigma=\sigma(\widetilde{d})$. 
1. With a compressive stress $\sigma\left(\widetilde{d}_{0}=-d_{0}\right)=-E_{1} m h_{s}<0$, the crack closes $\left(d_{0}+\widetilde{d}=0\right)$ and with $\sigma \leqslant-E_{1} m h_{s}<0$ it will not contribute to the elasticity of the medium.

2. With a strong crack opening $\left(d_{0}+\widetilde{d} \gg h_{s}\right)$, when $\frac{3 \pi E\left(d_{0}+\widetilde{d}\right)}{16\left(1-\nu_{0}^{2}\right) R} \gg E_{1}\left[d_{0}+\widetilde{d}+m h_{s}\right] \times$ $\times \exp \left(-\frac{d_{0}+\widetilde{d}}{h_{s}}\right)$, it follows from Eqs. (2.8) that $\sigma(\widetilde{d})=\frac{3 \pi E\left(d_{0}+\widetilde{d}\right)}{16\left(1-\nu_{0}^{2}\right) R}=\frac{3 \pi E \widetilde{d}}{16\left(1-\nu_{0}^{2}\right) R}>0$, i.e., the crack becomes linear and will not contribute to the nonlinear elasticity of the medium.

3 . The transition between the closed and open crack states will be smooth and nonlinear.

Thus, for a small strain, the dependence $\sigma=\sigma(\varepsilon)$ for a medium with cracks will be smooth (and nonlinear), and for large strains the medium will have bimodular properties. As a result, the equation of state of a medium with cracks will be close to a quadratic-bimodular (1.2).

Let us determine the dependence $\sigma=\sigma(\widetilde{d})$ in the quadratic approximation at the third stage of the crack deformation. Assuming in Eq. (2.8) that $|\sigma| \ll \sigma_{0}\left(d_{0}\right),|\widetilde{d}| \ll d_{0}, h_{s}$ we obtain

$$
\sigma(\widetilde{d})=\frac{\sigma_{0}}{\left(d_{0}+m h_{s}\right)}\left[\frac{m h_{s}\left(h_{s}+d_{0}\right)+d_{0}^{2}}{d_{0}}\left(\frac{\widetilde{d}}{h_{s}}\right)-\frac{d_{0}+(m-2) h_{s}}{2}\left(\frac{\widetilde{d}}{h_{s}}\right)^{2}\right] .
$$

From this equation, for $\left|\widetilde{d} / h_{s}\right| \ll \frac{2[1+m \xi(1+\xi)]}{1+(m-2) \xi}$, we find the expression for the change in the volume $\Delta V(\widetilde{d})$ of the crack:

$$
\Delta V(\widetilde{d})=\pi R^{2} \widetilde{d}(\sigma)=\alpha(\sigma / E)+(\beta / 2)(\sigma / E)^{2},
$$

where

$$
\xi=h_{s} / d_{0}, \quad \alpha=\frac{16\left(1-\nu^{2}\right) \xi R^{3}(1+m \xi)}{3[1+m \xi(1+\xi)]}, \quad \beta=\frac{256\left(1-\nu^{2}\right)^{2} \xi R^{4}[1+(m-2) \xi](1+m \xi)^{2}}{9 \pi d_{0}[1+m \xi(1+\xi)]^{3}} .
$$

From the equation of state (2.10) of a single crack, one can determine the equation of state of a solid body containing a system of such cracks. To obtain the equation of state of the medium, we consider, as an example, a rod with longitudinal stress $\sigma_{z z}=\sigma$ applied to its ends. For the rod without cracks, this stress creates a longitudinal strain [24, 25]:

$$
U_{z z}=\sigma / E
$$

The small element of the site $d S$ in the rod, the normal $\boldsymbol{n}$ to which is oriented at an angle $\varphi$ to the $z$ axis, will be acted upon by normal stress [24]: $\sigma_{n}=\sigma \cos ^{2} \varphi$. To determine the equation of state of a fractured medium, we introduce a new coordinate system $x_{i}^{\prime}$ (Fig. 2) and find an additional strain $d U_{z z}^{\prime}$ of the solid due to the presence of cracks, whose normal to the surface coincides with the axis $z^{\prime}$, and the projection of this normal to the plane $z=0$ makes an angle $\theta$ with the axis $x$. It is obvious that in such a medium there will be an additional strain along the axis $z^{\prime}$, caused by the opening of cracks [24]:

$$
d U_{z^{\prime} z^{\prime}}^{\prime}=\Delta V(\varphi) N(\varphi, \theta) \sin \varphi d \varphi d \theta
$$

where $N(\varphi, \theta)$ is the function of the distribution of cracks in the angles $\varphi$ and $\theta$, $N(\varphi, \theta) \sin \varphi d \varphi d \theta$ is the number of cracks with orientation from $\varphi$ and $\theta$ to $\varphi+d \varphi$, and $\theta+d \theta$, being in unit volume of the medium. All other components of the tensor $d U_{i^{\prime} k^{\prime}}^{\prime}$ will be zero. 


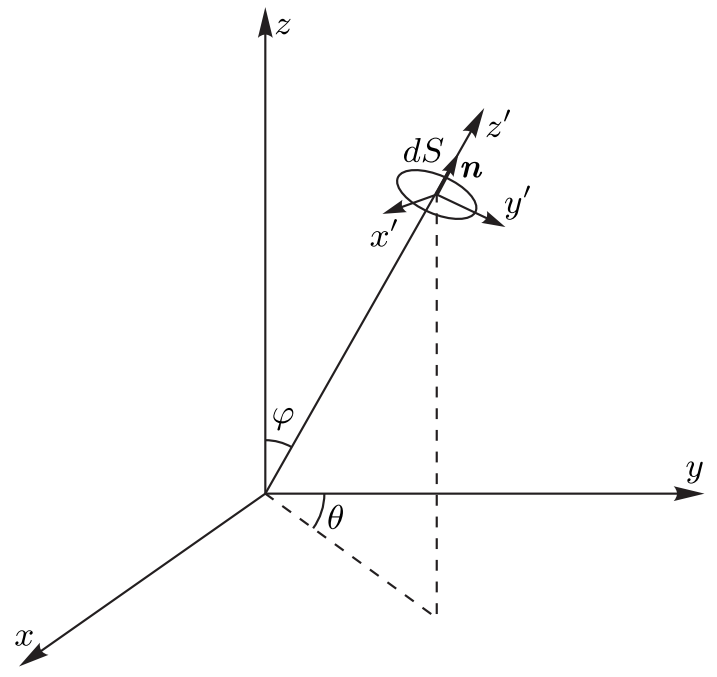

Fig. 2. Orientation of a single crack in coordinate systems $x, y, z$ and $x^{\prime}, y^{\prime}, z^{\prime}$.

Integrating this equation with respect to $\varphi$ and $\theta$ and taking into account the strain of the rod without cracks, we find the longitudinal strain $\varepsilon$ of the rod with cracks [21]:

$$
\varepsilon=\frac{\sigma}{E}\left(1+\int_{0}^{2 \pi} \int_{0}^{\pi / 2} N(\varphi, \theta) \sin \varphi \cos ^{4} \varphi\left[\alpha+\frac{\beta}{2} \frac{\sigma}{E} \cos ^{2} \varphi\right] d \varphi d \theta\right) .
$$

From (2.13) we find the equations of state of a medium with isotropically oriented cracks $\left[N(\varphi, \theta)=N_{0} / 2 \pi\right]$ corresponding to their different deformation modes:

$$
\sigma_{1}(\varepsilon)=E \varepsilon, \quad \sigma_{2}(\varepsilon)=\frac{E \varepsilon}{1+\mu}, \quad \sigma_{3}(\varepsilon)=\frac{E\left[\varepsilon-\Gamma \varepsilon^{2}\right]}{1+\alpha N_{0} / 5},
$$

where $\mu=\frac{16\left(1-\nu^{2}\right) N_{0} R^{3}}{15}, \Gamma=\frac{\beta N_{0}}{14\left[1+\left(\alpha N_{0} / 5\right)\right]^{2}}$.

Combining the equations of the state of the medium for the first and second stages of deformation of cracks, we obtain the equation of state with a bimodular nonlinearity at $\mu \ll 1$ :

$$
\sigma(\varepsilon)=\frac{E[1+(\mu / 2)]}{1+\mu}\left[\varepsilon-\frac{\mu}{2+\mu}|\varepsilon|\right] \cong E_{0}\left[\varepsilon-\frac{\mu}{2}|\varepsilon|\right],
$$

where $E_{0}=E[1-(\mu / 2)]$.

Now, combining Eq. (2.15) with the equation of state of the medium for the third stage of crack deformation (for $\alpha N_{0} / 5 \ll 1, \frac{8 N_{0} R^{3}\left(1-\nu^{2}\right)\left|1+(m-2) \mu-m \mu^{2}\right|}{15[1+m \mu(1+\mu)]} \ll 1$ ), we obtain the equation of state with quadratically-bimodular nonlinearity [7]:

$$
\sigma(\varepsilon) \cong E_{0}\left[\varepsilon-\frac{\gamma_{1} \varepsilon^{2}}{2\left(1+\gamma_{0}|\varepsilon|\right)}\right],
$$

where $\gamma_{1}=2 \Gamma, \gamma_{0}=\gamma_{1} / \mu=2 \Gamma / \mu$. 
We give estimates of the elastic characteristics of a solid body containing isotropically oriented cracks with the following parameters: the radius of cracks $R=10^{-1} \mathrm{~cm}$, $E=3 \cdot 10^{11} \mathrm{~g} / \mathrm{cm} \mathrm{s}^{2}, \nu_{0}=0.25$ the irregularities of the crack surfaces are in the form of obtuse cones with a solution angle $2 \psi=172^{\circ}$, the characteristic height of the unevenness is $h_{s}=10^{-6} \mathrm{~cm}, n=10^{3}, \sigma_{0}=5.6 \cdot 10^{5} \mathrm{~g} / \mathrm{cm} / \mathrm{s}, d_{0}=3 \cdot 10^{-7} \mathrm{~cm}, N_{0}=10 \mathrm{~cm}^{-3}$. In this case, the presence of cracks in the rod leads to a decrease in Young's modulus on $0.5 \%$, and the parameters of the nonlinearity are $\gamma_{1}=74, \gamma_{0}=7.4 \cdot 10^{3}, \mu / 2=\gamma_{1} / 2 \gamma_{0}=5 \cdot 10^{-3}$, respectively.

\section{Nonlinear wave equation}

Substituting the equation of state (1.2) into the equation of motion $\rho U_{t t}=\sigma_{x}(\varepsilon)$, and passing to the accompanying coordinate system, $\tau=t-x / C_{0}, x^{\prime}=x \geqslant 0[1,2]$, we obtain a single-wave equation for simple strain waves $\varepsilon(x, \tau)=U_{x}(x, \tau)$ :

$$
\frac{\partial \varepsilon}{\partial x}=-\frac{1}{4 C_{0}} \frac{\partial}{\partial \tau}\left(\frac{\gamma_{1} \varepsilon^{2}}{1+\gamma_{0}|\varepsilon|}\right),
$$

where $U$ is displacement, $\rho$ is density, $C_{0}=\left(E_{0} / \rho\right)^{1 / 2}$.

Generally speaking, the linear dissipative term $\eta \dot{\varepsilon}$ must also be taken into account in Eq. (1.2), where $\eta$ is the dissipation coefficient, but it can be neglected if we consider sufficiently strong and slow perturbations for which the inequality $E_{0}\left|\frac{\gamma_{1} \varepsilon^{2}}{2\left(1+\gamma_{0}|\varepsilon|\right)}\right| \gg \eta|\dot{\varepsilon}|[2,3]$ holds. In this case, it is possible to obtain exact analytic solutions of equation (3.1).

We reduce the wave equation (3.1) to the dimensionless form

$$
\frac{\partial e}{\partial z}=-\frac{\partial}{\partial \theta}\left(\frac{e^{2}}{1+a|e|}\right),
$$

where $e=\varepsilon / \varepsilon_{0}, \theta=\omega \tau, z=\gamma_{1} \varepsilon_{0} \omega x / 4 C_{0}, a=\gamma_{0} \varepsilon_{0} \geqslant 0, \varepsilon_{0}$ and $\omega$ being the characteristic amplitude and frequency of the wave. For definiteness, we will assume that $\gamma_{1}>0$.

\section{Propagation of a unipolar pulse}

Let us consider the propagation of a unipolar pulse, for which the boundary condition is given in the form

$$
e(0, \theta)=2 \cdot\left\{\begin{aligned}
\theta, & 0 \leqslant \theta \leqslant 1 / 2 \\
1-\theta, & 1 / 2 \leqslant \theta \leqslant 1,
\end{aligned}\right.
$$

where $\theta=\tau / T, T$ is the duration of the disturbance.

The exact solution of Eq. (3.2) with the boundary condition (4.1) is written in implicit form:

$$
e(z, \theta)=2 \cdot\left\{\begin{aligned}
\theta-\frac{e(z, \theta)[2+a e(z, \theta)]}{4[1+a e(z, \theta)]^{2}} z, & 0 \leqslant \theta \leqslant \theta_{m}(z) ; \\
1-\theta+\frac{e(z, \theta)[2+a e(z, \theta)]}{4[1+a e(z, \theta)]^{2}} z, & \theta_{m}(z) \leqslant \theta \leqslant 1,
\end{aligned}\right.
$$

where $e(z, \theta)=\varepsilon(z, \theta) / \varepsilon_{0}, a=\gamma_{0} \varepsilon_{0}, z=y \varepsilon_{0}, y=\gamma_{1} x / C_{0} T$. 

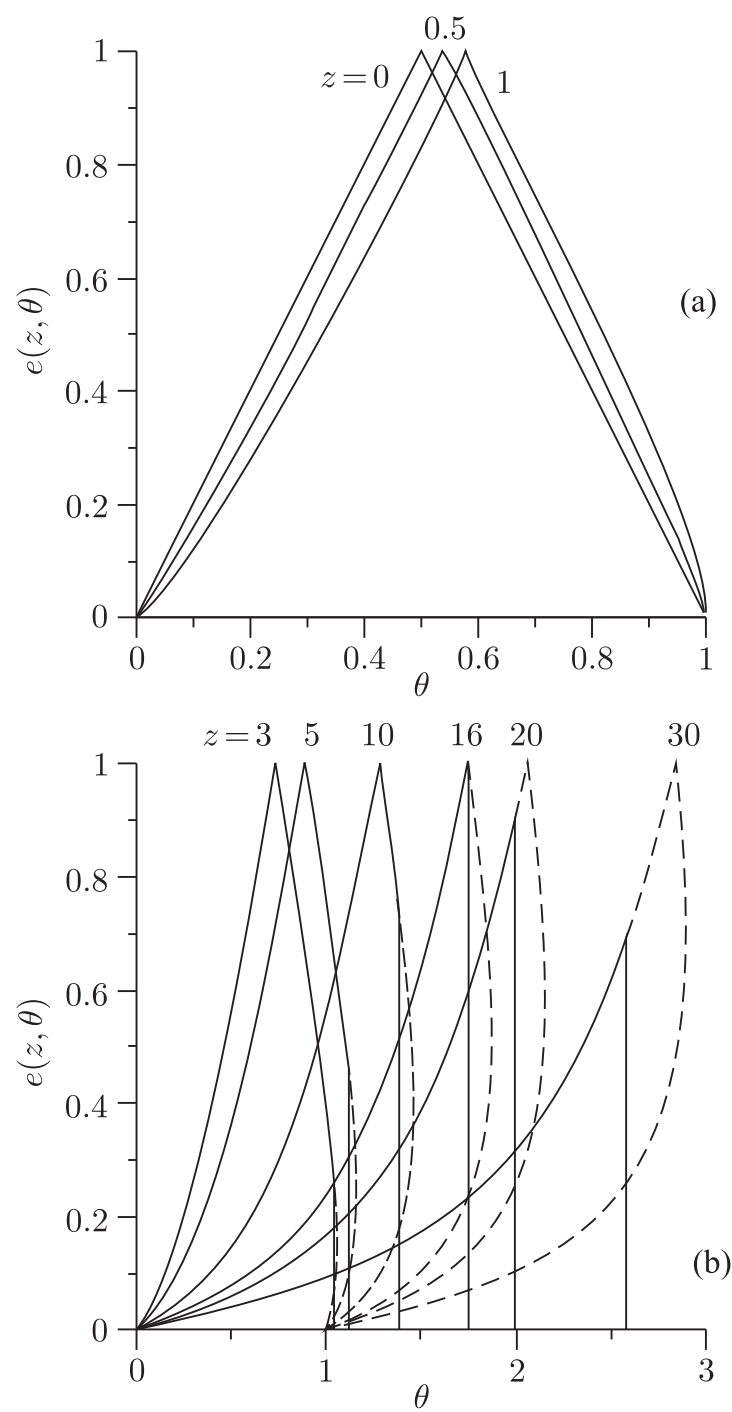

Fig. 3. The evolution of a unipolar pulse: (a) before the formation of a discontinuity, (b) after the formation of a discontinuity. The dashed lines show the parts of the pulse cut off by discontinuities.

The evolution of the pulse (4.2) at $a=3$ is shown in Fig. 3. With $z \leqslant 1$ its leading front $\left[e_{\theta}(z, \theta)>0\right]$ becomes "concave", and the rear one $\left[e_{\theta}(z, \theta)<0\right]$ becomes "convex" (Fig. 3a), while the amplitude and duration of the pulse remain equal to 1 , and the coordinate of its vertex is determined by the expression: $\theta_{m}(z)=\frac{1}{2}+\frac{(2+a) z}{4(1+a)^{2}}$. With $z=1$ the arrival of the pulse front (at the point $e=0, \theta=1$ ) becomes infinitely steep, and then (at $1<z \leqslant z^{*}=(1+a)^{2}$ ) a physically unrealizable ambiguity is formed in profile - an "overlap" (Fig. 3b), which, as in a quadratic medium [1-4], is eliminated by introducing at the point $\theta^{*}(z)=1+(\sqrt{z}-1)^{2} / 4 a$ a break determined from the conditions of "equality of areas" [1]:

$$
S(z)=\int_{0}^{\theta^{*}(z)} e(z, \theta) d \theta=\frac{1}{2} .
$$

The amplitude of this discontinuity is $e^{*}(z)=(\sqrt{z}-1) / a$. 
After the formation of the discontinuity (at $1<z \leqslant z^{*}=(1+a)^{2}$ ), the pulse amplitude is also equal 1 , and its duration $\theta^{*}(z)=1+(\sqrt{z}-1)^{2} / 4 a$ increases.

When $z>z^{*}=(1+a)^{2}$, the shape of the pulse, its amplitude $e_{m}(z)=e^{*}(z)$ and duration $\theta^{*}(z)$ are determined by the expressions

$$
\begin{gathered}
e(z, \theta)=2 \cdot\left\{\begin{array}{rr}
\theta-\frac{e(z, \theta)[2+a e(z, \theta)]}{4[1+a e(z, \theta)]^{2}} z, & 0 \leqslant \theta \leqslant \theta^{*}(z) \\
0, & \theta \leqslant 0, \quad \theta>\theta^{*}(z)
\end{array}\right. \\
e_{m}^{2}\left[1+\frac{z}{\left(1+a e_{m}\right)^{2}}\right]=2, \quad \theta^{*}(z)=\frac{e_{m}}{2}+\frac{z+1-2 / e_{m}^{2}}{4 a} .
\end{gathered}
$$

When $z \gg 1$ and $a e_{m} \ll 1$, we get $e_{m}(z)=\sqrt{2 / z}, \theta^{*}(z)=\sqrt{z / 2}$.

\section{Propagation of a periodic bipolar wave}

Let us now consider the propagation of a periodic, bipolar sawtooth wave, the boundary condition for one of its periods is given in the form

$$
e(0, \theta)=2 \cdot\left\{\begin{aligned}
\theta, & -1 / 2 \leqslant \theta \leqslant 1 / 2 \\
1-\theta, & 1 / 2 \leqslant \theta \leqslant 3 / 2,
\end{aligned}\right.
$$

where $\theta=\tau / T, T$ is the period of the wave.

The exact solution of Eq. (3.2) with the boundary condition (5.1) is written in implicit form:

$$
e(z, \theta)=2 \cdot\left\{\begin{aligned}
\theta-\frac{e(z, \theta)[2+a e(z, \theta)]}{4[1+a|e(z, \theta)|]^{2}} z, & \theta_{m}^{-}(z)-2 \leqslant \theta \leqslant \theta_{m}^{+}(z), \\
1-\theta+\frac{e(z, \theta)[2+a e(z, \theta)]}{4[1+a|e(z, \theta)|]^{2}} z, & \theta_{m}^{+}(z) \leqslant \theta \leqslant \theta_{m}^{-}(z),
\end{aligned}\right.
$$

where $e(z, \theta)=\varepsilon(z, \theta) / \varepsilon_{0}, a=\gamma_{0} \varepsilon_{0}, z=y \varepsilon_{0}, y=\gamma_{1} x / C_{0} T, \theta_{m}^{ \pm}(z)$ are the coordinates of the "vertices" of the wave, $\theta_{m}^{+}(z)=\frac{1}{2}+\frac{(2+a) z}{4(1+a)^{2}}, \theta_{m}^{-}(z)=\frac{3}{2}-\frac{(2+a) z}{4(1+a)^{2}}$.

Unlike a unipolar pulse whose duration varies during propagation, the period of the polarized periodic wave (5.2) is given by the boundary condition (5.1) and remains constant: $\theta_{0}=2$. The evolution of the wave (5.2) with $a=3$ is shown in Fig. 4. Here, as in the case of a unipolar pulse, with $z \leqslant 1$, the leading wave front $\left[e_{\theta}(z, \theta)>0\right]$ becomes "concave" and the rear wave front $\left[e_{\theta}(z, \theta)<0\right]$ becomes "convex" (Fig. 4a), while the amplitude and duration of the wave remain equal to 1 . With $z=1$ a wave front $e_{\theta}(z, \theta)<0$ (at a point, $e=0, \theta=1$ ) becomes infinitely steep, and further (Fig. 4b), with $1<z \leqslant z^{*}=(1+a)^{2}$, in it a "overlap" is formed, which is eliminated by introducing at the point $\theta^{*}(z)=1$ of discontinuity, determined from the condition of "equality of areas" [1-4]:

$$
S(z)=\int_{0}^{\theta^{*}(z)} e(z, \theta) d \theta=0 .
$$

The amplitude of the discontinuity is $e^{*}(z)=\frac{z-4+\sqrt{z(z+8)}}{4 a}$, where $z \leqslant \frac{2(1+a)^{2}}{2+a}$. 

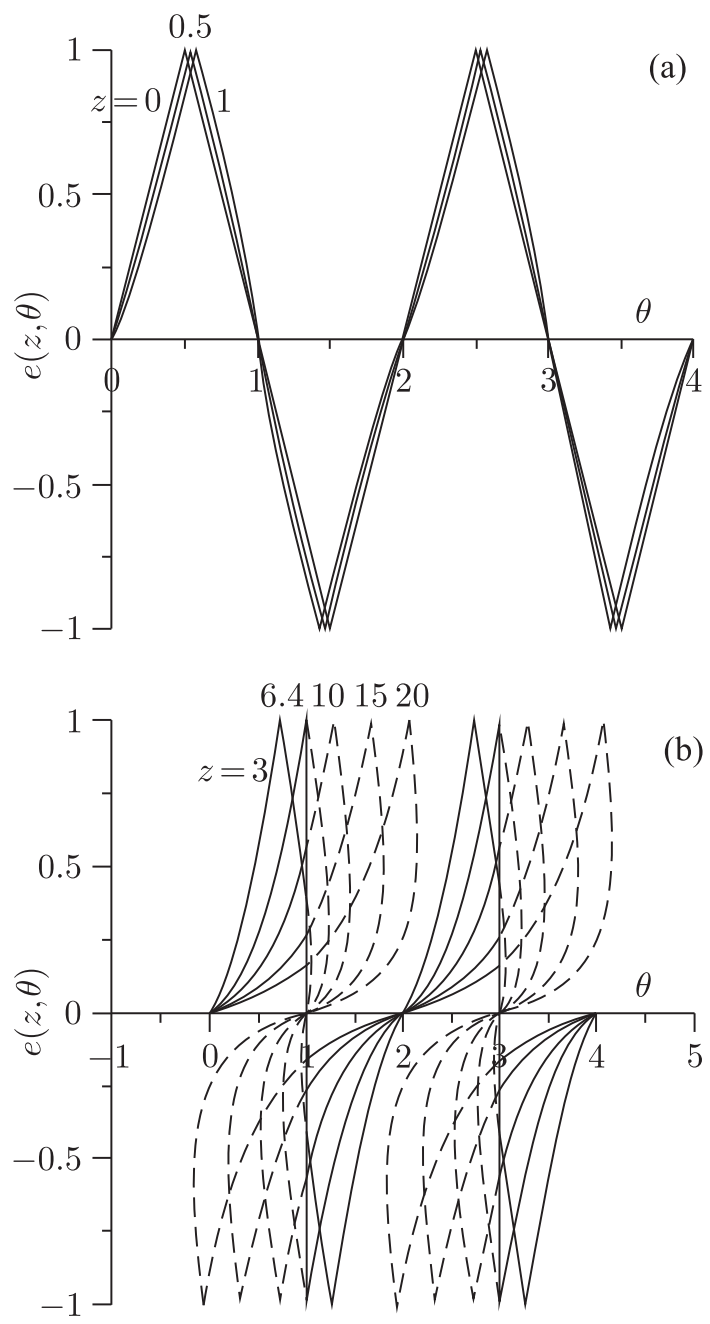

Fig. 4. Evolution of the sawtooth wave: (a) before the formation of the rupture, (b) after the formation of the rupture. The dashed lines show the parts of the wave that are cut off by discontinuities.

After the formation of the discontinuity $\left(\right.$ at $\left.z \leqslant \frac{2(1+a)^{2}}{2+a}\right)$, the amplitude of the wave is also equal to 1 , the coordinate of the discontinuity $\theta^{*}=1$.

When $z>\frac{2(1+a)^{2}}{2+a}$, the form of wave and its amplitude $e_{m}(z)=e^{*}(z)$ are determined by the expressions

$$
\begin{gathered}
e(z, \theta)=2 \cdot\left\{\begin{aligned}
\theta-\frac{e(z, \theta)[2+a e(z, \theta)]}{4[1+a|e(z, \theta)|]^{2}} z, & 0 \leqslant \theta \leqslant 1 ; \\
\theta-2-\frac{e(z, \theta)[2-a e(z, \theta)]}{4[1+a|e(z, \theta)|]^{2}} z, & 1 \leqslant \theta \leqslant 2,
\end{aligned}\right. \\
\frac{e_{m}}{2}+\frac{z}{4 a}\left[1-\frac{1}{\left(1+a e_{m}\right)^{2}}\right]=1 .
\end{gathered}
$$

When $z \gg 1$ and $a e_{m} \ll 1$, we get $e_{m}(z)=2 / z$. 


\section{Conclusion}

The equation of state of a micro-inhomogeneous medium with cracks, which has nonanalytic quadratically-bimodular elastic nonlinearity for longitudinal stress and strain, has been obtained. For a small strain, such a nonlinearity corresponds to a quadratic nonlinearity (as in the fiveconstant theory of elasticity). For a large strain, it corresponds to bimodular nonlinearity. Within the framework of the quadratically-bimodular equation of state, the wave evolutionary equation obtained and the propagation and evolution of single and periodic elastic waves in such media have been studied. Exact solutions have been obtained and numerical and graphical analysis of the evolution of the initially triangular unipolar perturbation and the periodic bipolar sawtooth wave has been carried out.

The main differences between the regularities of the propagation of a single unipolar perturbation (4.1) and a periodic bipolar wave (5.1) are as follows. The area under the curve $e=e(z, \theta)$ remains for both single disturbance and periodic wave. However, in the first case, the duration of a single disturbance (4.2) in the medium $(z>0)$ is not fixed and varies as $z$ increases: if $z \gg 1$, then $\theta^{*}(z)=\sqrt{z / 2}$. In the second case, the period of the periodic wave (5.1), (5.2) is strictly fixed and does not change. For this reason, the asymptotic expressions (for $z \gg 1$ ) for the amplitudes of a single disturbance and periodic wave are different. In the first case $e_{m}(z)=\sqrt{2 / z}$, in the second case $e_{m}(z)=2 / z$.

The results obtained are of interest for the development of the theory of nonlinear wave processes in media with nonanalytic nonlinearity; they can also be used to create nonlinear methods of acoustic diagnostics of structurally heterogeneous media and structural materials. In the future it will be interesting to consider nonlinear waves in such media with allowance for dissipation, dispersion, and relaxation.

\section{References}

[1] Landau, L. D. and Lifshitz, E. M., Course of Theoretical Physics: In 10 Vols.: Vol. 6. Fluid Mechanics, 2nd ed., Oxford: Butterworth-Heinemann, 2003.

[2] Rudenko, O.V. and Soluyan, S.I., Theoretical Foundations of Nonlinear Acoustics, New York: Springer, 2013.

[3] Naugolnykh, K.A. and Ostrovsky, L.A., Nonlinear Wave Processes in Acoustics, Cambridge Texts Appl. Math., Cambridge: Cambridge Univ. Press, 1998.

[4] Ryskin, N. M. and Trubezkov, D. I., Nonlinear Waves, Moscow: Fizmatlit, 2000 (Russian).

[5] Isakovich, M. A., General Acoustics, Moscow: Nauka, 1973 (Russian).

[6] Guyer, R.A. and Johnson, P.A., Nonlinear Mesoscopic Elasticity: Behaviour of Granular Media including Rocks and Soil, Weinheim: Wiley/VCH, 2009.

[7] Nazarov, V.E. and Radostin, A.V., Nonlinear Acoustic Waves in Micro-Inhomogeneous Solids, Chichester: Wiley, 2015.

[8] Ambartsumyan, S.A. and Khachatryan, A. A., The Different-Modules Theory of Elasticity, Mech. Solids, 1966, no. 1, pp. 29-34; see also: Inzh. Zh. Mekh. Tverd. Tela, 1966, no. 6, pp.64-67.

[9] Beresnev, I. A. and Nikolaev, A. V., Experimental Investigations of Nonlinear Seismic Effects, Phys. Earth Planet. Inter., 1988, vol.50, no. 1, pp. 83-87.

[10] Benveniste, Y., One-Dimensional Wave Propagation in Materials with Different Moduli in Tension and Compression, Int. J. Eng. Sci., 1980, vol. 18, no. 6, pp. 815-827.

[11] Maslov, V.P. and Mosolov, P. P., General Theory of the Solutions of the Equations of Motion of an Elastic Medium of Different Moduli, J. Appl. Math. Mech., 1985, vol.49, no. 3, pp.322-336; see also: Prikl. Mat. Mekh., 1985, vol.49, no.3, pp.419-437. 
[12] Nazarov, V.E. and Sutin, A. M., Harmonic Generation in the Propagation of Elastic Waves in Nonlinear Solid Media, Sov. Phys. Acoust., 1989, vol.35, no.4, pp.410-413; see also: Akust. Zh., 1989, vol. 35, no. 4, pp. 711-716.

[13] Nazarov, V. E. and Ostrovsky, L. A., Elastic Waves in Media with Strong Acoustic Nonlinearity, Sov. Phys. Acoust., 1990, vol.36, no. 1, pp.57-60; see also: Akust. Zh., 1990, vol. 36, no. 1, pp. $106-110$.

[14] Gavrilov, S. N. and Herman, G. C., Wave Propagation in a Semi-Infinite Heteromodular Elastic Bar Subjected to a Harmonic Loading, J. Sound Vibration, 2012, vol. 331, no. 20, pp. 4464-4480.

[15] Radostin, A., Nazarov, V., and Kiyashko, S., Propagation of Nonlinear Acoustic Waves in Bimodular Media with Linear Dissipation, Wave Motion, 2013, vol. 50, no. 2, pp. 191-196.

[16] Nazarov, V.E., Kiyashko, S. B., and Radostin, A. V., Self-Similar Waves in Media with Bimodular Elastic Nonlinearity and Relaxation, Nelin. Dinam., 2015, vol.11, no. 2, pp. 209-218 (Russian).

[17] Nazarov, V.E., Kiyashko, S. B., and Radostin, A. V., The Wave Processes in Micro-Inhomogeneous Media with Different-Modulus Nonlinearity and Relaxation, Radiophys. Quantum El., 2016, vol.59, no. 3, pp. 246-256; see also: Izv. Vyssh. Uchebn. Zaved. Radiofizika, 2016, vol. 59, no. 3, pp. 275-285.

[18] Rudenko, O. V., Inhomogeneous Burgers Equation with Modular Nonlinearity: Excitation and Evolution of High-Intensity Waves, Dokl. Math., 2017, vol.95, no.3, pp.291-294; see also: Dokl. Akad. Nauk, 2017, vol.474, no.6, pp.671-674.

[19] Rudenko, O. V., Modular Solitons, Dokl. Math., 2016, vol.94, no.3, pp.708-711; see also: Dokl. Akad. Nauk, 2016, vol.471, no. 6, pp.651-654.

[20] Hedberg, C. M. and Rudenko, O. V., Collisions, Mutual Losses and Annihilation of Pulses in a Modular Nonlinear Medium, Nonlinear Dynam., 2017, vol. 90, no. 3, pp. 2083-2091.

[21] Nazarov, V. E. and Sutin, A. M., Nonlinear Elastic Constants of Solids with Cracks, J. Acoust. Soc. Am., 1997, vol. 102, no. 6, pp. 3349-3354.

[22] Johnson, K. L., Contact Mechanics, Cambridge: Cambridge Univ. Press, 1985.

[23] Sneddon, I. N., Fourier Transformations, New York: McGraw-Hill, 1951.

[24] Timoshenko, S. P. and Goodier, J. N., Theory of Elasticity, 3rd ed., New York: McGraw-Hill, 1970.

[25] Landau, L. D. and Lifshitz, E. M., Course of Theoretical Physics: Vol. 7. Theory of Elasticity, 3rd ed., Oxford: Butterworth/Heinemann, 1986.

[26] Mathews, J. and Walker, R. L., Mathematical Methods of Physics, 2nd ed., Reading, Mass.: AddisonWesley, 1971. 